\title{
One Giant Leap for Pharmacology
}

\author{
Sara Eyal ' (D) $\cdot$ Hartmut Derendorf ${ }^{2}$
}

Received: 18 February 2020 / Accepted: 18 February 2020 / Published online: 2 March 2020

(C) Springer Science+Business Media, LLC, part of Springer Nature 2020

Although astronauts are usually healthy and in good physical shape, they still can become sick, get infected or even develop blood clots. During a recent study on jugular vein flow and morphology, obstructive thrombosis was detected for the first time in an astronaut in space $(1,2)$. Due to limited availability of anticoagulants onboard the International Space Station (ISS) the astronaut was initially treated with enoxaparin with dose reduction thirty three days later to allow treatment extension. Meanwhile, apixaban was delivered to the ISS by a supply aircraft. Apixaban was administered to the astronaut from 42 days after the diagnosis until four days before return to Earth. Spontaneous flow was still absent after 90 days of apixaban treatment onboard the ISS but was shown on landing (1). The authors of this report highlighted challenges of space medicine, including novel medical conditions with no evidence for decision making, the need in telemedicine, and limited pharmacy. This point was also illustrated in Scott Kelly's autobiography Endurance: My Year in Space, A Lifetime of Discovery. The author, a retired NASA astronaut who participated in NASA's Twin Study, describes severe leg swelling, orthostatism, nausea, rash, and pain within days after landing. Self-treating his symptoms with ibuprofen, he explains "no one in the hospital will have seen symptoms of having been in space for a year" (3).

In this Theme issue of Pharmaceutical Research, dedicated to drugs in space, Eyal and Derendorf (4) describe current

Guest Editors: Sara Eyal and Hartmut Derendort

Sara Eyal

sarae@ekmd.huji.ac.il

Institute for Drug Research, School of Pharmacy, Faculty of Medicine Hebrew University of Jerusalem, Ein Kerem, Jerusalem, Israel

2 Department of Pharmaceutics, University of Florida, Gainesville, Florida 32610-0494, USA knowledge and uncertainties of drug pharmacokinetics and pharmacodynamics during spaceflight and return to Earth. Back to the above mentioned case of thromboembolism, the efficacy of apixaban and enoxaparin treatment onboard the ISS is actually unknown. Drug bioavailability, concentrations of plasma albumin and coagulation factors (and clot properties), activity of drug metabolizing enzymes and drug transporters, and renal function, could all have been affected by the microgravity of spaceflight. A related manuscript by Berman and Eyal highlights an additional aspect of pharmacological treatment in space, drug-drug interactions (5). The authors analyzed lists of medications available to astronauts onboard the ISS and report on a potential for multiple interactions, such as commutative sedation or antimuscarinic drug effects. Such interactions are likely, for instance, when astronauts selfcombine two medications for sleep disorders, as was described before (6).

With challenges come opportunities, too: the microgravity of space provides a novel set of conditions for drug R\&D. In a special commentary, Giulianotti and Low (7) introduce the ISS as a unique platform for research that helps NASA and partners reduce the risk of human exploration of deep space and enables discoveries with applications to life on Earth. The commentary describes improved crystallization of low molecular weight compounds and macromolecules, research in rodent models, and microfluidic systems (tissue chips) as accelerated models of human disease. Also presented is a recent partnership between the NIH's Center for Advanced Translational Science (NCATS) and The ISS National Lab which allowed the launch and use of microfluidic platforms onboard the ISS.

Low and Giulianotti (8) further describe the ongoing nine projects of the Tissue Chips in Space initiative that investigate alterations in the immune and musculoskeletal systems, renal and cardiac function, and barriers of the gut and the brain. Those systems are a key for mechanistic understanding of the physiological changes that occur in the human body during 


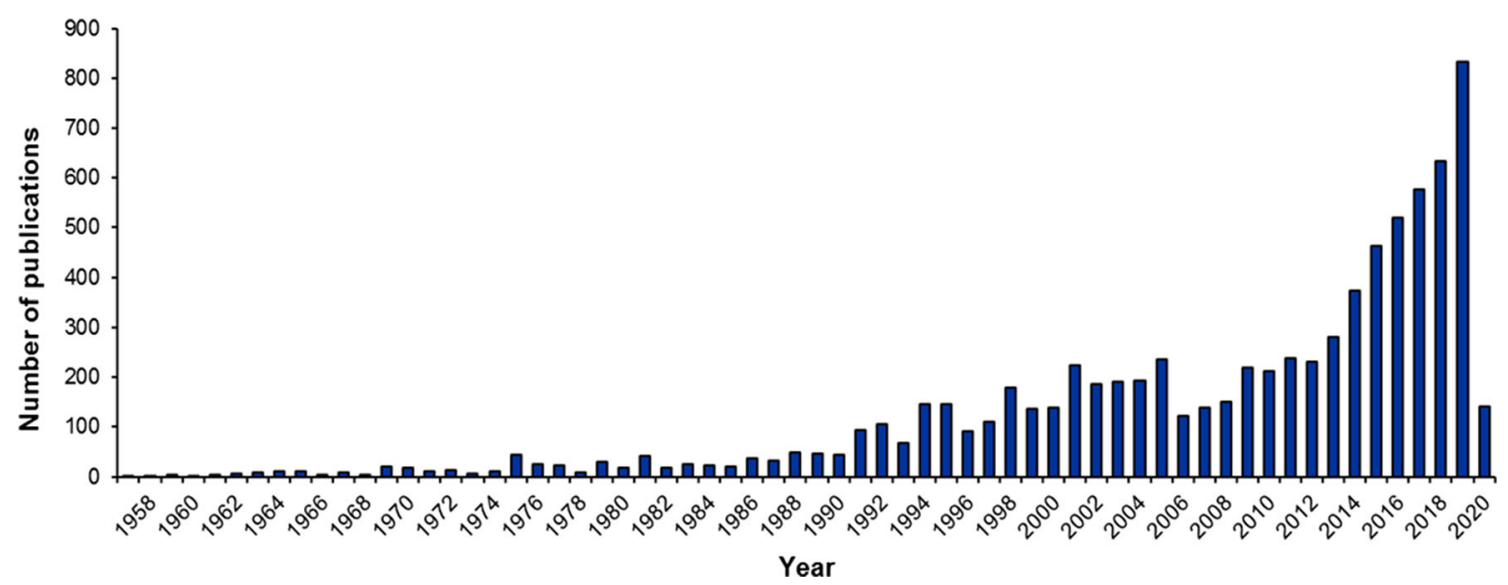

Fig. I Number of citations per year retrieved for the query Astronaut* in PubMed.

spaceflight without the confounding factors of animal experimentation. Insights gained through this project have multiple potential applications, including those relevant for the pharmaceutical industry. Also described are the challenges associated with experimentation in space and the contribution of implementation partners that developed miniaturized labs, adapted to conform to NASA flight safety specifications.

An industry point of view on pharmaceutical research in space is presented by Shimon Amselem (9), who reviews traditional and emerging platforms for conducting studies in microgravity conditions. Looking to the near future, this manuscript highlights innovative, unmanned platforms and experimental minilabs that can operate onboard the ISS or on nanosatellites while omitting the use in astronaut-related resources. Such systems can transmit information obtained by microscopy and spectroscopy to Earth thus allowing the researcher to intervene with the experimental conditions based on real time data. In addition, studies conducted on the ISS can be retrieved for further analyses at the researcher's lab. These systems can significantly broaden the opportunities for conducting research in space by academia and industry.

There are several ways in which space can advance pharmacology and pharmaceutical sciences for the benefit of both space travelers and patients on Earth. A new discipline in pharmacology develops as human presence in space expands, e.g., with the initiation of Axiom Space's trips to a habitable commercial module that will be attached the ISS (10) and the launching of space hotels into orbit (11). Space tourist are expected to be older and have more medical conditions than career astronauts. In addition, exploration missions to deep space, e.g., to the Moon and Mars, will significantly enhance radiation exposure and pose new challenges to the human body. Current principles for medical treatment in space are in many ways similar to those practiced on the ground decades ago. It is imperative that we gain a broad appreciation of the impact of microgravity and cosmic radiation on drug stability, efficacy and safety. We have to make sure that we have a better understanding of what drugs and what doses should be used during spaceflight. Currently, it is assumed that drug therapy in space will be the same as on Earth with little experimental evidence. It is time to perform some actual pharmacokinetic and pharmacodynamic studies in space to shed more light on this subject and assure optimum therapeutic care of the astronauts.

At the same time, new insights can be gained through research onboard the ISS, unmanned vehicles, and satellites. Developments already achieved in space include Amgen's denosumab (Prolia) (12) and new crystalline suspensions of Merck's pembrolizumab (Keytruda) (13). The interest of the scientific and medical community in space keeps growing (Fig. 1), and the technology is there. It is time to harness space for identifying new drug targets, generate novel formulations, and even produce drugs in space.

\section{ACKNOWLEDGMENTS AND DISCLOSURES}

No financial support was received for this study. Sara Eyal is on a sabbatical leave at SpacePharma, Israel, from July 1st 2019. Sara Eyal is affiliated with the David R. Bloom Centre for Pharmacy and Dr. Adolf and Klara Brettler Centre for Research in Molecular Pharmacology and Therapeutics at The Hebrew University of Jerusalem, Israel. Hartmut Derendorf is Distinguished Professor Emeritus of Pharmaceutics at the University of Florida and has served on the Nutrition and Therapeutics Committee of the NASA Space Medicine Program as well as on the NASA Exploration Medical Capabilities Element's Pharmacy Project Expert Review Panel.

\section{REFERENCES}

1. Aunon-Chancellor SM, Pattarini JM, Moll S, Sargsyan A. Venous thrombosis during spaceflight. N Engl J Med. 2020;382:89-90.

2. Marshall-Goebel K, Laurie SS, Alferova IV, Arbeille P, AunonChancellor SM, Ebert DJ, et al. Assessment of jugular venous blood flow stasis and thrombosis during spaceflight. JAMA Netw Open. 2019;2:e1915011. 
3. Kelly S. Endurance: my year in space, a lifetime of discovery. New York: Vintage; Reprint edition; 2018.

4. Eyal S, Derendorf H. Medications in space: in search of a pharmacologist's guide to the galaxy. Pharm Res. 2019;36:148.

5. Berman E, Eyal S. Drug interactions in space: a cause for concern? Pharm Res. 2019;36:114.

6. Barger LK, Flynn-Evans EE, Kubey A, Walsh L, Ronda JM, Wang $\mathrm{W}$, et al. Prevalence of sleep deficiency and use of hypnotic drugs in astronauts before, during, and after spaceflight: an observational study. Lancet Neurol. 2014;13:904-12.

7. Giulianotti MA, Low LA. Pharmaceutical research enabled through microgravity: perspectives on the use of the International Space Station U.S. National Laboratory. Pharm Res. 2019;37:1.

8. Low LA, Giulianotti MA. Tissue chips in space: modeling human diseases in microgravity. Pharm Res. 2019;37:8.

9. Amselem S. Remote controlled autonomous microgravity lab platforms for drug research in space. Pharm Res. 2019;36:183.

10. National Aeronautics and Space Agency. NASA selects first commercial destination module for International Space Station. https://www.nasa.gov/press-release/nasa-selects-first-commercialdestination-module-for-international-space-station. Updated Feb 5 2020. Accessed Feb 52020.

11. McFall-Johnsen, M. At least 3 companies plan to launch space hotels into orbit, offering inflatable rooms, 16 sunsets per day, and even space Quidditch. Business Insider. Published: Nov 9 2019. Accessed: Feb 52020.

12. National Aeronautics and Space Administration. Health \& Medicine: NASA Spinoff: Rodent Research Contributes to Osteoporosis Treatments. Available from https://spinoff.nasa. gov/Spinoff2016/hm 1.html. 2016. Accessed August 292019.

13. Reichert P, Prosise W, Fischmann TO, Scapin G, Narasimhan G, Spinale A, et al. Pembrolizumab microgravity crystallization experimentation. NPJ Microgravity. 2019;5:28.

Publisher's Note Springer Nature remains neutral with regard to jurisdictional claims in published maps and institutional affliations. 